\title{
Electric-field-driven octahedral rotation in perovskite
}

\author{
Wonshik Kyung $\mathbb{D}^{1,2,3,7}$, Choong H. Kim $\mathbb{D}^{1,2,7}$, Yeong Kwan Kim ${ }^{4}$, Beomyoung Kim ${ }^{3}$, Chul Kim ${ }^{5}$, Woobin Jung ${ }^{1,2}$, Junyoung Kwon ${ }^{1,2}$,

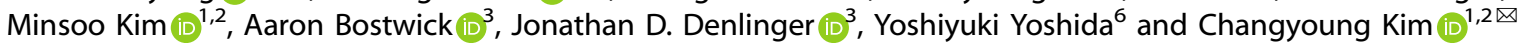

Rotation of $\mathrm{MO}_{6}(\mathrm{M}=$ transition metal) octahedra is a key determinant of the physical properties of perovskite materials. Therefore, tuning physical properties, one of the most important goals in condensed matter research, may be accomplished by controlling octahedral rotation (OR). In this study, it is demonstrated that OR can be driven by an electric field in $\mathrm{Sr}_{2} \mathrm{RuO}_{4}$. Rotated octahedra in the surface layer of $\mathrm{Sr}_{2} \mathrm{RuO}_{4}$ are restored to the unrotated bulk structure upon dosing the surface with $\mathrm{K}$. Theoretical investigation shows that $\mathrm{OR}$ in $\mathrm{Sr}_{2} \mathrm{RuO}_{4}$ originates from the surface electric field, which can be tuned via the screening effect of the overlaid $\mathrm{K}$ layer. This work establishes not only that variation in the OR angle can be induced by an electric field, but also provides a way to control OR, which is an important step toward in situ control of the physical properties of perovskite oxides.

npj Quantum Materials (2021)6:5; https://doi.org/10.1038/s41535-020-00306-1

\section{INTRODUCTION}

Perovskite materials possess some of the most interesting properties in condensed matter physics, such as superconductivity, metal-insulator transitions, and ferroicity ${ }^{1}$. Theoretical and experimental research has proven that octahedral rotation (OR) plays an important role in those properties. For instance, OR significantly affects metal-insulator transitions ${ }^{2,3}$ and exotic orbital-selective phenomena ${ }^{4,5}$ by changing the inter-site electron hopping probability and even the structural symmetry of materials. Furthermore, the magnetic ground state of a material is often governed by OR through altered super-exchange or Dzyaloshinskii-Moriya interactions ${ }^{6}$. Therefore, the ability to readily vary OR would be an important step toward controlling such physical properties.

However, in spite of its importance, there are a limited number of reports on controlling $\mathrm{OR}^{7-9}$. The main reason for this is that the OR angle is thought to be an inherent characteristic of a material, determined by the steric effect arising from the sizes of its constituent atoms ${ }^{10,11}$. Thus, most of the attempts to control OR have been limited to substitution of atoms with different ionic sizes or application of strains using different substrates. However, these methods do not truly allow control of the OR angle as they cannot be applied in situ, and typically cause complex side effects $^{12,13}$.

When attempting to control physical properties through the OR angle, various parameters should be considered, such as pressure and magnetic and electric fields. Among these parameters, the electric field has distinct advantages in terms of convenience, controllability, and minimal power consumption. In this letter, we report electric-field-dependent evolution of the OR angle on the surface of $\mathrm{Sr}_{2} \mathrm{RuO}_{4}$. By means of in situ dosing with potassium (K), the surface electric field can be tuned through the screening effect of the overlying $\mathrm{K}$ atoms. $\mathrm{Sr}_{2} \mathrm{RuO}_{4}$ was chosen as the target material given its distinct surface layer-driven bands, which arise due to differences in its structural symmetry (a finite OR angle at its surface and no rotation of its bulk $)^{14-16}$; these properties not only provide clean surfaces, but also make investigation of its electronic structure relatively easy. Our results obtained using the surface-sensitive techniques of low-energy electron diffraction (LEED) and angle-resolved photoelectron spectroscopy (ARPES) indicate that reduction of the electric field results in a reduced OR angle (down to zero). Our density functional theory (DFT) studies have shown that the surface electric potential (or surface electric field) ${ }^{17,18}$ is responsible for the OR on the surface of $\mathrm{Sr}_{2} \mathrm{RuO}_{4}$, which in turn implies the possibility of controlling the OR angle via an electric field.

\section{RESULTS}

Disappearance of $\mathrm{Sr}_{2} \mathrm{RuO}_{4}$ surface states upon $\mathrm{K}$ coverage

Figure 1 shows Fermi surface (FS) maps of freshly cleaved and Kdosed $\mathrm{Sr}_{2} \mathrm{RuO}_{4}$ surfaces. The main features are two bulk electron pockets $\left(\beta^{b}\right.$ and $\left.\gamma^{b}\right)$ centered at $\Gamma$ and a bulk hole pocket $\left(a^{b}\right)$ centered at $(\pi, \pi)$. In addition to these bulk FSs, we detected additional FSs from the surface $\left(\alpha^{s}, \beta^{s}, \gamma^{s}\right)$ and zone-folded surface $\left(a^{\text {sf }}, \beta^{\text {sf }}, \gamma^{\text {sf }}\right)$ bands (Fig. 1a), consistent with previously reported ARPES results ${ }^{15,16,19}$. Notably, when we deposit $K$ on the surface with coverage above one monolayer (ML), all of the surfacerelated FSs disappear (Fig. 1b), which is somewhat similar to the case of surface aging of $\mathrm{Sr}_{2} \mathrm{RuO}_{4}$ (refs. ${ }^{16,19}$ ). To deduce the reason for this disappearance, we systematically varied the coverage of $\mathrm{K}$ while conducting LEED and ARPES measurements.

LEED is one of the most direct and convenient methods to investigate in-plane crystal symmetry. In case when there is no OR distortion, only the $1 \times 1$ peak should appear. When the in-plane OR angle is finite, the unit cell is transformed to a $(\sqrt{2} \times \sqrt{2}) R 45^{\circ}$ unit cell, and consequently additional fractional peaks $(\sqrt{2} \times \sqrt{2})$ should appear in the LEED pattern. The relative intensity of the fractional to integer spot is approximately proportional to the OR angle $\mathrm{e}^{20,21}$. The LEED results from our systematic investigation of $K$ coverage are shown in Fig. 2a. In the LEED data of pristine $\mathrm{Sr}_{2} \mathrm{RuO}_{4}$, we can observe both $1 \times 1$ and $\sqrt{2} \times \sqrt{2}$ peaks. As the $\mathrm{K}$ coverage increases, the OR-driven $\sqrt{2} \times \sqrt{2}$ peaks gradually weaken and eventually disappear above $1 \mathrm{ML}$ of $\mathrm{K}$, while the $1 \times 1$ peaks remain robust. Therefore, we suspect that the $\mathrm{K}$ layer

\footnotetext{
${ }^{1}$ Center for Correlated Electron Systems, Institute for Basic Science (IBS), Seoul 08826, Republic of Korea. ${ }^{2}$ Department of Physics and Astronomy, Seoul National University (SNU), Seoul 08826, Republic of Korea. ${ }^{3}$ Advanced Light Source, Lawrence Berkeley National Laboratory, Berkeley, CA 94720, USA. ${ }^{4}$ Department of Physics, KAIST, Daejeon 34141, Republic of Korea. ${ }^{5}$ Institute of Physics and Applied Physics, Yonsei University, Seoul 03722, Korea. ${ }^{6}$ National Institute of Advanced Industrial Science and Technology, Tsukuba 305-8568, Japan. ${ }^{7}$ These authors contributed equally: Wonshik Kyung, Choong H. Kim. ${ }^{\circledR}$ email: changyoung@snu.ac.kr
} 
gradually and eventually completely suppresses the $\mathrm{OR}$ of the surface $\mathrm{Sr}_{2} \mathrm{RuO}_{4}$.

The ARPES results from our systematic investigation of $\mathrm{K}$ coverage are shown in Fig. $2 \mathrm{~b}$; they also provide evidence for the suppression of OR in the surface $\mathrm{Sr}_{2} \mathrm{RuO}_{4}$. The data for pristine $\mathrm{Sr}_{2} \mathrm{RuO}_{4}$ along $\Gamma-\mathrm{M}-\Gamma$ (Fig. 2b) show $\beta^{\mathrm{b}}, \beta^{\mathrm{s}}, \gamma^{\mathrm{b}}$, and $\gamma^{\mathrm{s}}$ bands. As the $\mathrm{K}$ coverage increases, the $\beta^{\mathrm{s}}$ band, instead of being suppressed,

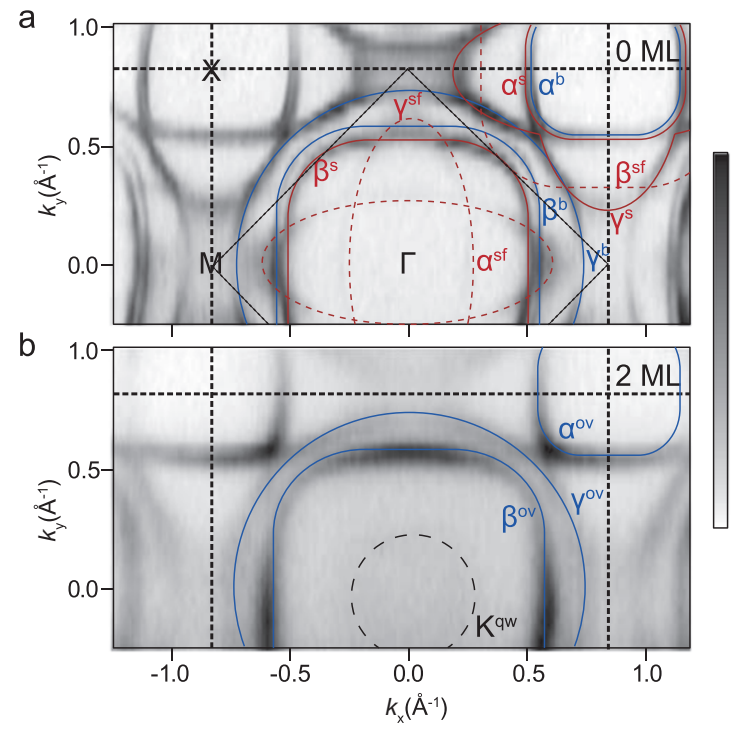

Fig. 1 Fermi surface (FS) maps of $\mathrm{Sr}_{2} \mathrm{RuO}_{4}$. a Fresh $\mathrm{Sr}_{2} \mathrm{RuO}_{4}$ and b $\mathrm{Sr}_{2} \mathrm{RuO}_{4}$ covered in two monolayers (MLs) of $\mathrm{K}$. The black thick (thin) dashed lines mark the Brillouin zone of the bulk (surface) $\mathrm{Sr}_{2} \mathrm{RuO}_{4}$ without (with) octahedral rotation. $\mathrm{RuO}_{6}$ octahedra on the surface rotate, resulting in a $\sqrt{2} \times \sqrt{2}$ reconstructed surface that causes replica FSs to appear. Superscripts $b, s$, sf, and ov denote bulk, surface, surface folding, and overlap, respectively. The blue (red) guidelines indicate the bulk (surface) bands. $\mathrm{K}^{\mathrm{qw}}$ indicates a $\mathrm{K}$ circular quantum well state (Supplementary Fig. 4). moves toward $\beta^{\mathrm{b}}$ before finally merging with it. As for the $\gamma$ bands, $\gamma^{\mathrm{b}}$ remains the same while the spectral weight of the $\gamma^{\mathrm{s}}$ band at the M-point gradually weakens. These observations indicate that the difference between the bulk and surface electronic structures gradually decreased, consistent with the suppression of OR in the surface layer. Our detailed analysis regarding the ARPES spectra (Supplementary Fig. 1) also supports that the suppression of the surface layer-driven bands is induced by suppression of the surface OR.

\section{Cause of the suppressed OR}

The next question is why the OR angle reduces with $\mathrm{K}$-dosing. Surface alkali metal atoms can play roles in electron doping, chemical bonding, and changing the surface electric potential; we shall consider each in turn. First, we consider the possibility that the vanishing $O R$ is caused by electron transfer from the alkali metal to the $\mathrm{Sr}_{2} \mathrm{RuO}_{4}$. To investigate this, we obtained the electron occupations of the bands from their FS volume; we list these in the Supplementary Table 1 along with all other values discussed here. We find that there is an occupation difference of 0.09 electrons between the fresh surface (3.82) and that with $1 \mathrm{ML}$ of $\mathrm{K}$ (3.91), which agrees well with the number (0.07) extracted from our theoretical study (Supplementary Fig. 2). However, an average transfer of 0.09 electrons from the $\mathrm{K}$ atoms is not likely to cause complete suppression of OR considering the case of $(\mathrm{Sr}, \mathrm{La})_{2} \mathrm{RuO}_{4}$, which is regarded as electron-doped $\mathrm{Sr}_{2} \mathrm{RuO}_{4}$ (Supplementary Fig. 3). On the other hand, effects arising from chemical bonding cannot provide an adequate explanation either; our measured $\mathrm{K} 3 p$ core-level spectra as a function of $\mathrm{K}$ coverage (Supplementary Fig. 4) clearly show the absence of chemical bonding associated with $\mathrm{K}$ atoms. Furthermore, as mentioned, our experimental and theoretical results (Supplementary Table 1 and Supplementary Fig. 2) suggest that there an electron transfer of only $0.07-0.09$ from the $\mathrm{K}$ atoms, which is too small to be due to chemical bonding between the $\mathrm{K}$ and $\mathrm{O}$ atoms.

As the change in OR angle cannot be attributed to electron doping or chemical bonding effects, let us now consider the gradient of the surface electric potential. In general, the electric potential is modulated due to the electric potential difference

a
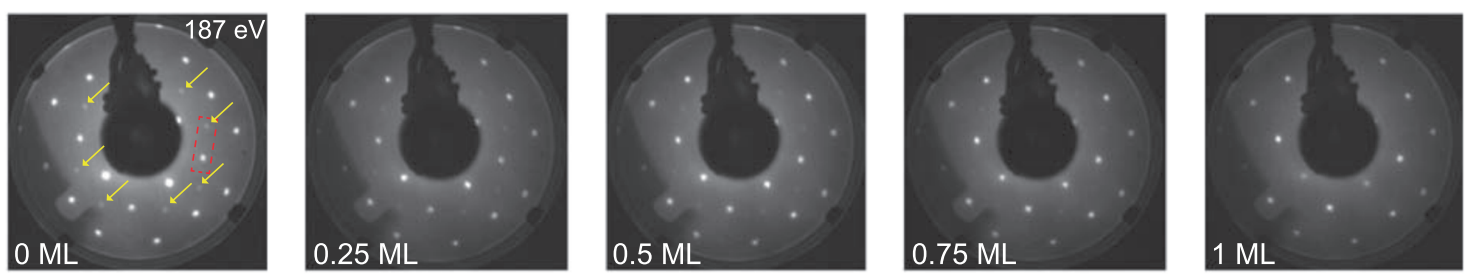

b
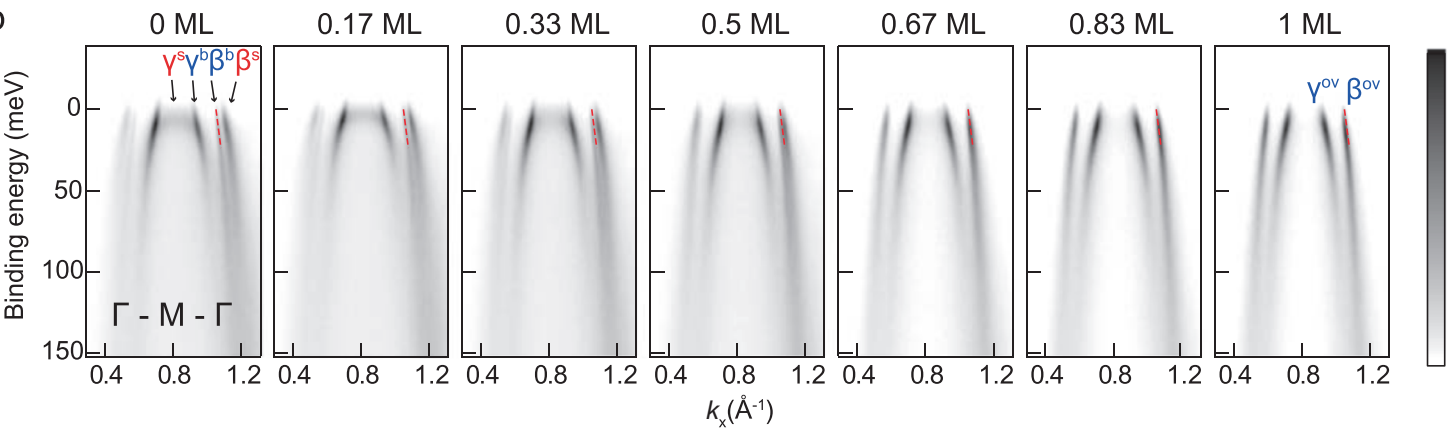

Fig. 2 Low-energy electron diffraction and angle-resolved photoelectron spectroscopy results according to the $\mathrm{K}$ coverage. a Electron diffraction images for various $\mathrm{K}$ coverages $(0,0.25,0.5,0.75$, and $1 \mathrm{ML})$. The yellow arrows indicate peaks due to $\sqrt{2} \times \sqrt{2}$ surface reconstruction; as the $\mathrm{K}$ coverage increases, these peaks gradually become weaker and eventually disappear. The rectangle in red is the region of quantitative intensity analysis in Supplementary Fig. 1. b Photoelectron spectroscopy images along $\Gamma-\mathrm{M}-\Gamma$ for various $\mathrm{K}$ coverages $(0,0.17$, $0.34,0.5,0.67,0.83$, and $1 \mathrm{ML})$. Red dashed lines are to mark the position of $\beta^{\mathrm{b}}$ of $0 \mathrm{ML}$ coverage. 
a
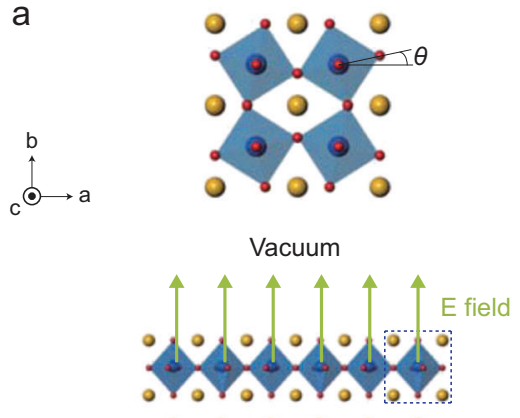

०.0.0,0.0,0

$0.0 .090 .0 \%$

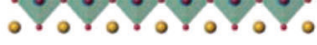

$0,0,0,0,0,0$

0.0 .0 .0 .0 .0

- $\mathrm{Sr} \quad \mathrm{Ru}$

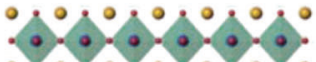
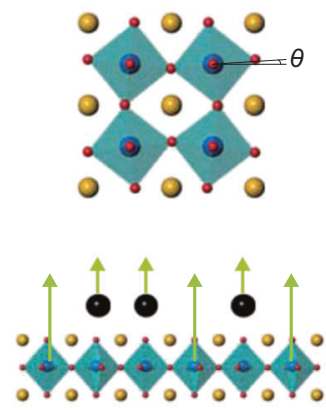

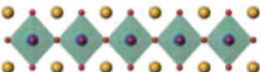

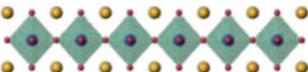

$0,0,0,0,0,0$

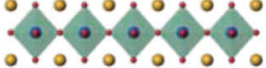

- $\mathrm{C} \mathrm{k}$

b

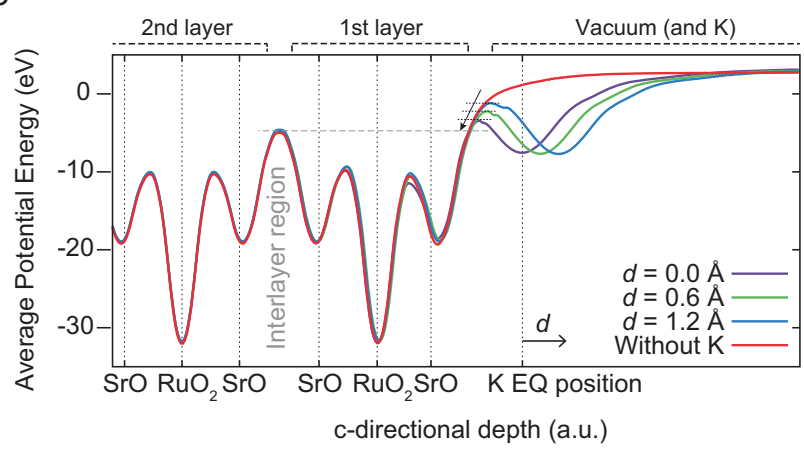

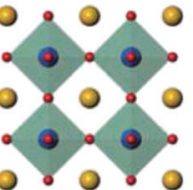

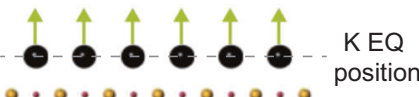

position
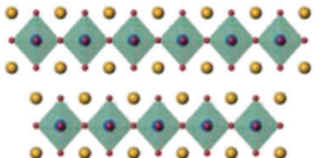

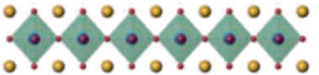

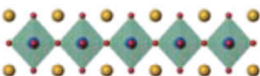

C

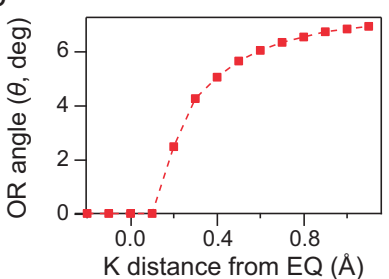

d

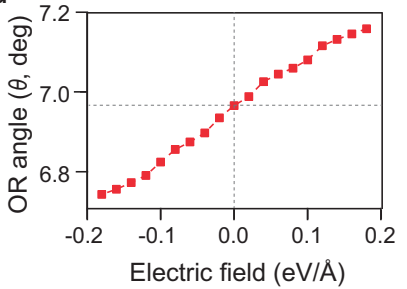

Fig. 3 Electric-potential-driven octahedral rotation (OR). a Crystal structure of $\mathrm{Sr}_{2} \mathrm{RuO}_{4}$ with three different $\mathrm{K}$ coverages (left: fresh, middle: partial coverage, right: $1 \mathrm{ML}$ ). The dashed rectangle marks the area for the enlarged view in Fig. 4a. b-d Density functional theory (DFT) results for a slab of $\mathrm{Sr}_{2} \mathrm{RuO}_{4}$ five layers thick. b Electric potential energy as a function of the depth in the c-direction (surface normal), for various $\mathrm{K}$ layer distances from the equilibrium position: $d=0.0,0.6,1.2 \AA$ and without a $\mathrm{K}$ layer. The equilibrium position of the surface $\mathrm{K}$ layer (9.4 $\AA$ from the second outermost Ru-O layer) was obtained from the DFT calculation. The gray horizontal dashed line indicates the electric potential energy in the $\mathrm{Sr}_{2} \mathrm{RuO}_{4}$ interlayer region. As the K-layer distance decreases, the electric potential in the SrO-K region gradually decreases toward the value of the interlayer region (black arrow). c Plot of OR angle as a function of the K-layer distance from the equilibrium position. d Plot of OR angle as a function of electric field.

between vacuum and solid. This electric potential modulation is greatly affected by the surface condition, as illustrated in Fig. 3a and $b$. To investigate how electric potential relates to the OR angle, we performed a DFT calculation which has been widely used to describe low-energy physics in $\mathrm{Sr}_{2} \mathrm{RuO}_{4}$ (refs. ${ }^{15,16,22}$ ), by constructing a five-layer slab of $\mathrm{Sr}_{2} \mathrm{RuO}_{4}$ with and without an $\mathrm{ML}$ of $\mathrm{K}$ overlaid. We also performed calculations for various K-layer distances away from the equilibrium position, to investigate the evolution of the electric potential and OR angle.

First, we note that the potential energy decreases gradually toward the value of the interlayer region as the $\mathrm{K}$ layer approaches the equilibrium position (the arrow in Fig. 3b). This suggests that the role of the $\mathrm{K}$ layer is to mitigate the surface electric field; $1 \mathrm{ML}$ of $\mathrm{K}$ causes the electric potential in the surface region to become similar to the interlayer potential. Consequently, the surface layer is in a bulk-like potential, which should lead to the suppression of OR (Fig. 3a). The calculated OR angle monotonically evolves from zero to fully rotated as the K-layer distance increases from the equilibrium position (Fig. $3 \mathrm{c}$ ). These observations strongly indicate that the origin of the OR is in the electric field, consistent with the experimental data in Fig. 2.

Even though it is not essential to prove the electric origin of the $\mathrm{OR}$, it is noteworthy that the OR evolution in the calculation in Fig. $3 c$ fairly consistently reproduces the behavior according to the thickness of the $\mathrm{K}$ layer seen in the experimental data. Partial Kcoverage cases have been simulated by artificially moving the $\mathrm{K}$ layer away from the equilibrium position ${ }^{23}$. The similarity between the experimental and theoretical results may be understood in the following way. Figure $3 \mathrm{~b}$ shows the calculated electric potential as a function of the K-layer distance from surface layer. The electric potential at the interface (between the outermost SrO layer and the $\mathrm{K}$ layer) monotonically and gradually changes as the K-layer distance changes. We expect that the surface electric field will be screened by $\mathrm{K}$ atoms proportionally with the K coverage (Fig. 3a). Therefore, the 'moving K-layer distance' should generate similar trends in the electric potential as does the $\mathrm{K}$ coverage. Our electronic structure with 'moving K-layer distance' does indeed show trends consistent with our K-coverage-dependent ARPES 
results (Supplementary Fig. 5); hence, the method should be reasonable to observe the overall trend.

To directly investigate the role of the electric field in the OR angle, we performed another five-layer slab calculation, this time with an external electric field applied perpendicular to the surface (Fig. 3d). Our DFT calculations of total energy predict that the surface OR changes, exhibiting behavior proportional to the increase of $\sim 0.15^{\circ}$ per $0.1 \mathrm{~V} \AA^{-1}$ (Fig. 3d); thus, the electric field appears to be coupled to the OR. Therefore, we conclude that the electric field is responsible for the OR in the $\mathrm{Sr}_{2} \mathrm{RuO}_{4}$ surface layer and thus the OR can be varied by tuning the electric potential.

\section{Mechanism of the electric-field-driven OR}

The next step is to find out how an electric field couples with the OR. Previous theoretical studies have shown that ferroelectric-like atomic displacement competes with the OR, which is the reason why most of ferroelectric materials do not have OR distortion ${ }^{24}$. Therefore, it is natural to consider non-uniform atomic displacements driven by depth-dependent electric field as the cause for the OR angle change. From our DFT calculation with K-layer distance variation, we extract atomic displacement in the outermost $\mathrm{Sr}-\mathrm{O}$ layer. It shows that the distance between upper and lower $\mathrm{Sr}$ atoms in the surface $\mathrm{Sr}_{2} \mathrm{RuO}_{4}$ layer is the most sensitive factor to the K-layer distance (Supplementary Fig. 6). It is found that the vertical $\mathrm{Sr}-\mathrm{Sr}$ distance (defined in Fig. 3a) increases with the electric field (K-layer distance) and changes more than $0.1 \AA$ as shown in Fig. $4 a$.

In order to check whether the (vertical) $\mathrm{Sr}$-Sr distance is coupled to the $\mathrm{OR}$ angle variation, we have estimated $\mathrm{OR}$ angle from bulk $\mathrm{Sr}_{2} \mathrm{RuO}_{4}$ calculation as a function of the $\mathrm{Sr}-\mathrm{Sr}$ distance (Fig. 4b). As can be seen in Fig. 4b, variation of the $\mathrm{Sr}$-Sr distance successfully reproduces emergence of the OR angle in $\mathrm{Sr}_{2} \mathrm{RuO}_{4}$ in the range over which the $\mathrm{Sr}-\mathrm{Sr}$ distance varies in Fig. $4 \mathrm{a}$. Therefore, it appears that the Sr-Sr distance is the mediating parameter between the surface electric field and OR angle. As the stronger (weaker) surface electric field makes a larger (smaller) $\mathrm{Sr}-\mathrm{Sr}$ distance, and it eventually leads to a larger (smaller) OR angle. In this context, we can reconsider how OR varies depending on the situation in $\mathrm{Sr}_{2} \mathrm{RuO}_{4}$. Absence of surface electric potential makes octahedron unrotated in bulk $\mathrm{Sr}_{2} \mathrm{RuO}_{4}$. On the other hand, the surface layer feels the surface electric potential, and thus the (surface electric potential driven) large $\mathrm{Sr}-\mathrm{Sr}$ distance makes octahedron rotated in the surface layer. Upon $\mathrm{K}$ dosing, the $\mathrm{K}$ atoms gradually reduce the surface electric potential toward the value of bulk electric potential. Then, the OR angle in the surface layer gradually decreases down to zero as we observed.

This mechanism can be applied to various materials other than $\mathrm{Sr}_{2} \mathrm{RuO}_{4}$. We performed additional bulk calculation on $\mathrm{Sr}_{2} \mathrm{RhO}_{4}$
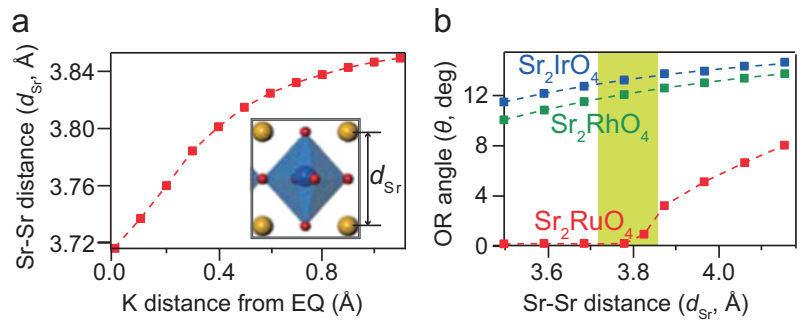

Fig. 4 Vertical Sr-Sr distance as the link between the surface electric potential and OR angle. a DFT calculation results from a five- $\mathrm{Sr}_{2} \mathrm{RuO}_{4}$-layer slab. The plot shows the $\mathrm{Sr}-\mathrm{Sr}$ distance as a function of the K-layer distance. Inset: enlarged view of an octahedron in the surface $\mathrm{Sr}_{2} \mathrm{RuO}_{4}$ layer. $\mathrm{Sr}-\mathrm{Sr}$ distance $\left(d_{\mathrm{Sr}}\right)$ is defined as the vertical distance between upper and lower $\mathrm{Sr}$ atoms. b DFT results for the OR angle as a function of the $\mathrm{Sr}$-Sr distance for bulk $\mathrm{Sr}_{2} \mathrm{RuO}_{4}, \mathrm{Sr}_{2} \mathrm{RhO}_{4}$, and $\mathrm{Sr}_{2} \mathrm{IrO}_{4}$. The shaded region marks the actual range over which the $\mathrm{Sr}-\mathrm{Sr}$ distance varies in $\mathbf{a}$. and $\mathrm{Sr}_{2} \mathrm{IrO}_{4}$ for various $\mathrm{Sr}-\mathrm{Sr}$ distances (Fig. $4 \mathrm{~b}$ ), and it is found that OR angles of $\mathrm{Sr}_{2} \mathrm{RhO}_{4}$ and $\mathrm{Sr}_{2} \mathrm{IrO}_{4}$ are affected by $\mathrm{Sr}-\mathrm{Sr}$ distance in the same manner as in the case of $\mathrm{Sr}_{2} \mathrm{RuO}_{4}$, even though the large initial OR angles of $\mathrm{Sr}_{2} \mathrm{RhO}_{4}$ and $\mathrm{Sr}_{2} \mathrm{IrO}_{4}$ make their variation less dramatic. These results not only consistently simulate the $\mathrm{OR}$ angle variation behavior in $\mathrm{K}$-dosed $\mathrm{Sr}_{2} \mathrm{RuO}_{4}$, but also suggest that there might be universal coupling between the $\mathrm{OR}$ angle and cation distance in transition-metal oxide (TMO).

\section{DISCUSSION}

We shall now discuss the difference between the local and external electric fields to allow our result to be more fully understood. Even though we theoretically demonstrated an OR angle change by an external electric field (Fig. $3 \mathrm{~d}$ ), $\sim 4 \vee \AA^{-1}$ is required for full suppression of the $O R$, which is not practical. However, a local electric field (potential gradient near the surface) can induce full suppression of the OR in $\mathrm{Sr}_{2} \mathrm{RuO}_{4}$, as shown in Fig. $3 \mathrm{~b}$, where varying the K-layer distance changes the electric potential energy on the order of a few eV. This case demonstrates the advantages of exploiting the local electric field, in terms of strength and controllability. In practice, such local electric fields could be achieved by using special techniques such as ionic gating or even exploiting existing, naturally occurring fields at interfaces. In that respect, issues in groups of material heterostructures, which exhibit numerous exotic phenomena that the corresponding bulk crystals do not, such as superconductivity ${ }^{25,26}$, metal-insulator transitions ${ }^{27}$, and controllable ferromagnetism could be revisited ${ }^{28}$. We suspect that local electric fields at interfaces play a significant role in generating these phenomena, and our findings should provide important clues regarding their microscopic mechanisms.

Since OR is not a polar distortion, whereas an electric field would produce a polar distortion, an electric field effect has rarely been considered when determining the OR angle. In this regard, the discovery of hybrid improper ferroelectricity (HIF) was a surprise because it shows an unexpected coupling between $O R$ and ferroelectric (polar) distortion of $\mathrm{Ca}$ atom (A-atom in $\mathrm{A}_{3} \mathrm{~B}_{2} \mathrm{O}_{7}$ ) in double-layer perovskites such as $\mathrm{Ca}_{3} \mathrm{Ti}_{2} \mathrm{O}_{7}$ (refs. ${ }^{29,30}$ ). Some theoretical studies have proposed electrical control of physical properties by changing the OR angle via the HIF mechanism ${ }^{31,32}$, but this has not yet been achieved experimentally. Contrary to the case of $\mathrm{HIF}_{2} \mathrm{Sr}_{2} \mathrm{RuO}_{4}$ is a metal and normally is not a suitable candidate for ferroelectric distortion. However, a finite displacement for $\mathrm{Sr}$ atom (A-atom in $\mathrm{A}_{2} \mathrm{BO}_{4}$ ) in the surface $\mathrm{Sr}_{2} \mathrm{RuO}_{4}$ layer can be induced by the surface electric potential, and then the $\mathrm{Sr}$ displacement may establish the connection between electric field and OR (Fig. 4). Note that A-atom mediates electric field and OR in both $\mathrm{HIF}$ and surface $\mathrm{Sr}_{2} \mathrm{RuO}_{4}$ cases, which may imply a possible universal mechanism of $\mathrm{OR}$ angle variation via $\mathrm{A}$-atom modulation. Our work not only is to show a change in OR angle via electric field, but also may initiate follow-up studies to elucidate the mechanism of OR angle variation.

Finally, let us discuss possible effects of OR angle variation in systems with strong electron correlation since perovskite oxides are typically in the strong electron correlation regime. Since OR angle can significantly affect the exchange interaction as well as electron localization, it is expected that change in the OR angle can lead to various phenomena, e.g., magnetism and metal-insulator transitions. Such effects may be found in the case of $\mathrm{Ca}_{2-x} \mathrm{Sr}_{x} \mathrm{RuO}_{4}$ (CSRO) which may be viewed as $\mathrm{Sr}_{2} \mathrm{RuO}_{4}$ with OR angle variation. In CSRO, rich and complex phases ${ }^{33-35}$, such as magnetisms (ferromagnetism ${ }^{33}$ or antiferromagnetism ${ }^{34}$ ), appear and emergence of those phenomena are attributed to OR distortions ${ }^{5,36,37}$. Another important aspect is that the electronic structure of $\mathrm{Sr}_{2} \mathrm{RuO}_{4}$ possesses a van Hove singularity near the Fermi level whose position is very sensitive to the OR angle. The large density of states from the van Hove singularity can 
boost the instability from electron correlation. This implies that we could effectively control the electron correlation strength of the system via the OR angle. Control of OR therefore may allow us a more diverse controllability of physical properties in strongly correlated materials.

In conclusion, our experimental and theoretical investigations demonstrate that variation of electric potential is responsible for the OR angle in the $\mathrm{Sr}_{2} \mathrm{RuO}_{4}$ surface layer, and that OR angle can be varied by tuning the electric potential through surface $K$ dosing. Our result not only sheds light on the mechanism of octahedral distortion found in various oxide systems but is also an important step toward electric field control of physical properties via variation of the $\mathrm{OR}$ angle in perovskite oxides.

\section{METHODS}

\section{ARPES and LEED measurement conditions}

ARPES ( $h v=70 \mathrm{eV}$ ) measurements were performed at beam lines (BLs) 4.0.3 and 7.0.2 of the Advanced Light Source, Lawrence Berkeley National Laboratory, USA. Potassium dosing was carried out by evaporating $\mathrm{K}$ onto the sample using commercial alkali metal dispensers (SAES). Spectra were acquired with R8000 (BL 4.0.3) and R4000 (BL 7.0.2) electron analyzers (Scienta). Total energy resolution was set to $12 \mathrm{meV}$, and the angular resolution was $0.00163 \AA^{-1}$. Cleaving and dosing of the samples were done at $20 \mathrm{~K}$ in an ultra-high vacuum better than $5 \times 10^{-11}$ Torr. LEED measurements were performed at BL 7.0.2 of the Advanced Light Source and at the Center for Correlated Electron Systems of the Institute for Basic Science, Seoul National University, Republic of Korea, using a LEED spectrometer (SPECS) with an electron energy of $187 \mathrm{eV}$.

\section{Information of DFT calculation}

As the feasibility of using DFT to understand the structural and electronic properties of $\mathrm{Sr}_{2} \mathrm{RuO}_{4}$ has been shown through previous studies ${ }^{15,16,22}$, we performed first-principle calculation using the non-spin-polarized DFT method without spin-orbit coupling. The Perdew-Burke-Ernzerhof form of the exchange-correlation functional was used as implemented in VASP software $^{38,39}$. We used a $600 \mathrm{eV}$ plane wave cut-off energy and $12 \times 12 \times 1$ $k$-points for all calculations and the projector augmented wave method. The in-plane lattice constant was fixed at the experimental value of $\mathrm{Sr}_{2} \mathrm{RuO}_{4}$. All the internal atomic positions were fully relaxed until the maximum force was below $0.5 \mathrm{meV}^{-1}$ while the symmetries of the system (point group $\mathrm{D}_{4 \mathrm{~h}}$ ) were maintained during the relaxation. In practice, since full relaxation is numerically unstable, we fixed the rotation angle of the octahedron and relaxed only the vertical positions of the atoms of the surface. In this way, the energy curve as a function rotation angle was obtained, and the angle with the energy minimum was found. We also checked that no additional symmetry lowering occurs even without symmetry constraints for a few cases. To mimic partial K coverage, we performed a five-layer slab calculation with $15 \AA$ vacuum layer, which is symmetric with respect to the middle layer with an overlying $\mathrm{K}$ atom layer. In this calculation, we relaxed the distance between the $\mathrm{K}$ layer and the $\mathrm{Sr}_{2} \mathrm{RuO}_{4}$ to find the equilibrium K-layer position. The resultant value was the 'K EQ position' in Fig. 3 ( $9.4 \AA^{-1}$ from the second Ru-O layer). In the calculation, we relaxed both the outermost layer and the K-layer distance, which explains why we defined the position relative to the second $\mathrm{Ru}-\mathrm{O}$ layer. The location of each $\mathrm{K}$ atom could not be specified experimentally, so we assumed it to be above the apical oxygen atom of the outermost $\mathrm{Sr}-\mathrm{O}$ layer (as illustrated in Fig. 3a) since that is the energetically most stable position in our DFT calculation. For the bulk calculations of $\mathrm{Sr}_{2} \mathrm{RhO}_{4}$ and $\mathrm{Sr}_{2} \mid \mathrm{rO}_{4}$, the in-plane lattice constants were also fixed as the experimental lattice constant of each compound, 5.4516 and $5.4956 \AA$, respectively. In these calculations, we fixed the $\mathrm{Sr}$ and Ir positions and allowed to move oxygen atoms only to find the optimum rotation angle of the octahedron for a given $\mathrm{Sr}-\mathrm{Sr}$ distance.

\section{DATA AVAILABILITY}

The datasets generated during and/or analyzed during the current study are available from the corresponding author upon request.
Received: 18 August 2020; Accepted: 12 December 2020; Published online: 08 January 2021

\section{REFERENCES}

1. Rao, C. N. R. Transition metal oxides. Annu. Rev. Phys. Chem. 40, 291-326 (1989).

2. Balachandran, P. V. \& Rondinelli, J. M. Interplay of octahedral rotations and breathing distortions in charge-ordering perovskite oxides. Phys. Rev. B 88 054101 (2013).

3. Nie, Y. F. et al. Interplay of spin-orbit interactions, dimensionality, and octahedral rotations in semimetallic SrlrO 3 . Phys. Rev. Lett. 114, 016401 (2015).

4. Kim, B. J. et al. Missing $x y$-band Fermi surface in $4 d$ transition-metal oxide $\mathrm{Sr}_{2} \mathrm{RhO}_{4}$ : effect of the octahedra rotation on the electronic structure. Phys. Rev. Lett. 97, 106401 (2006).

5. Ko, E., Kim, B. J., Kim, C. \& Choi, H. J. Strong orbital-dependent $d$-band hybridization and Fermi-surface reconstruction in metallic $\mathrm{Ca}_{2-x} \mathrm{Sr}_{x} \mathrm{RuO}_{4}$. Phys. Rev. Lett. 98, 226401 (2007).

6. Dzyaloshinsky, I. A thermodynamic theory of "weak" ferromagnetism of antiferromagnetics. J. Phys. Chem. Solids 4, 241-255 (1958).

7. Lu, W. et al. Control of oxygen octahedral rotations and physical properties in $\mathrm{SrRuO}_{3}$ films. Phys. Rev. B 88, 214115 (2013).

8. May, S. J. et al. Control of octahedral rotations in $\left(\mathrm{LaNiO}_{3}\right)_{n} /\left(\mathrm{SrMnO}_{3}\right)_{\mathrm{m}}$ superlattices. Phys. Rev. B 83, 153411 (2011).

9. Gao, R. et al. Interfacial octahedral rotation mismatch control of the symmetry and properties of $\mathrm{SrRuO}_{3}$. ACS Appl. Mater. Interfaces 8, 14871-14878 (2016).

10. Megaw, H. D. A simple theory of the off-centre displacement of cations in octahedral environments. Acta Crystallogr. B B24, 149-153 (1968).

11. Brown, I. D. Chemical and steric constraints in inorganic solids. Acta Crystallogr. $B$ B48, 553-572 (1992).

12. Cheng, S. L., Du, C. H., Chuang, T. H. \& Lin, J. G. Atomic replacement effects on the band structure of doped perovskite thin films. Sci. Rep. 9, 7828 (2019).

13. Steppke, A. et al. Strong peak in $\mathrm{T}_{\mathrm{c}}$ of $\mathrm{Sr}_{2} \mathrm{RuO}_{4}$ under uniaxial pressure. Science 355, eaaf9398 (2017)

14. Matzdorf, R. et al. Ferromagnetism stabilized by lattice distortion at the surface of the $p$-wave superconductor $\mathrm{Sr}_{2} \mathrm{RuO}_{4}$. Science 289, 746-748 (2000).

15. Damascelli, A. et al. Fermi surface, surface states, and surface reconstruction in $\mathrm{Sr}_{2} \mathrm{RuO}_{4}$. Phys. Rev. Lett. 85, 5194 (2000).

16. Veenstra, C. N. et al. Determining the surface-to-bulk progression in the normalstate electronic structure of $\mathrm{Sr}_{2} \mathrm{RuO}_{4}$ by angle-resolved photoemission and density functional theory. Phys. Rev. Lett. 110, 097004 (2013).

17. Ishikawa, K. \& Uemori, T. Surface relaxation in ferroelectric perovskites. Phys. Rev. B 60, 11841 (1999).

18. Barnes, S. E., leda, J. \& Maekawa, S. Rashba spin-orbit anisotropy and the electric field control of magnetism. Sci. Rep. 4, 4105 (2014).

19. Zabolotnyy, V. B. et al. Surface and bulk electronic structure of the unconventional superconductor $\mathrm{Sr}_{2} \mathrm{RuO}_{4}$ : unusual splitting of the $\beta$ band. N. J. Phys. 14, 063039 (2012).

20. Hu, B. et al. Surface and bulk structural properties of single-crystalline $\mathrm{Sr}_{3} \mathrm{Ru}_{2} \mathrm{O}_{7}$. Phys. Rev. B 81, 184104 (2010).

21. $\mathrm{Li}, \mathrm{G}$. et al. Atomic-scale fingerprint of $\mathrm{Mn}$ dopant at the surface of $\mathrm{Sr}_{3}\left(\mathrm{Ru}_{1-\mathrm{x}} \mathrm{Mn}_{\mathrm{x}}\right)_{2} \mathrm{O}_{7}$. Sci. Rep. 3, 2882 (2013).

22. Veenstra, $\mathrm{C}$. N. et al. Spin-orbital entanglement and the breakdown of singlets and triplets in $\mathrm{Sr}_{2} \mathrm{RuO}_{4}$ revealed by spin- and angle-resolved photoemission spectroscopy. Phys. Rev. Lett. 112, 127002 (2014).

23. Kim, J. et al. Observation of tunable band gap and anisotropic Dirac semimetal state in black phosphorus. Science 349, 723-726 (2015).

24. Benedek, N. A. \& Fennie, C. J. Why are there so few perovskite ferroelectrics? J. Phys. Chem. C 117, 13339-13349 (2013).

25. Reyren, N. et al. Superconducting interfaces between insulating oxides. Science 317, 1196-1199 (2007).

26. Aird, A. \& Salje, E. K. H. Sheet superconductivity in twin walls: experimental evidence of $\mathrm{WO}_{3-\mathrm{x}}$. J. Phys. Condens. Matter 10, L377-L380 (1998).

27. Meyers, D. et al. Pure electronic metal-insulator transition at the interface of complex oxides. Sci. Rep. 6, 27934 (2016).

28. Heron, J. T. et al. Electric-field-induced magnetization reversal in a ferromagnetmultiferroic heterostructure. Phys. Rev. Lett. 107, 217202 (2011).

29. Benedek, N. A. \& Fennie, C. J. Hybrid improper ferroelectricity: a mechanism for controllable polarization-magnetization coupling. Phys. Rev. Lett. 106, 107204 (2011).

30. Oh, Y. S. et al. Experimental demonstration of hybrid improper ferroelectricity and the presence of abundant charged walls in $(\mathrm{Ca}, \mathrm{Sr})_{3} \mathrm{Ti}_{2} \mathrm{O}_{7}$ crystals. Nat. Mater. 14, 407-413 (2015).

31. Benedek, N. A., Mulder, A. T. \& Fennie, C. J. Polar octahedral rotations: a path to new multifunctional materials. J. Solid State Chem. 195, 11-20 (2012). 
32. Liu, X. Q. et al. Hybrid improper ferroelectricity and possible ferroelectric switching paths in $\mathrm{Sr}_{3} \mathrm{Hf}_{2} \mathrm{O}_{7}$. J. Appl. Phys. 125, 114105 (2019).

33. Nakatsuji, S. et al. Heavy-mass Fermi liquid near a ferromagnetic instability in layered ruthenates. Phys. Rev. Lett. 90, 137202 (2003).

34. Carlo, J. P. et al. New magnetic phase diagram of $(\mathrm{Sr}, \mathrm{Ca})_{2} \mathrm{RuO}_{4}$. Nat. Mater. 11, 323-328 (2012)

35. Gorelov, E. et al. Nature of the Mott Transition in $\mathrm{Ca}_{2} \mathrm{RuO}_{4}$. Phys. Rev. Lett. 104, 226401 (2010).

36. Noh, H.-J. et al. Electronic structure and evolution of the orbital state in metallic $\mathrm{Ca}_{2-x} \mathrm{Sr}_{x} \mathrm{RuO}_{4}$. Phys. Rev. B 72, 052411 (2005).

37. Neupane, M. et al. Observation of a novel orbital selective Mott transition in $\mathrm{Ca}_{1.8} \mathrm{Sr}_{0.2} \mathrm{RuO}_{4}$. Phys. Rev. Lett. 103, 097001 (2009).

38. Kresse, G. \& Furthmüller, J. Efficient iterative schemes for ab initio total-energy calculations using a plane-wave basis set. Phys. Rev. B 54, 11169 (1996).

39. Kresse, G. \& Joubert, D. From ultrasoft pseudopotentials to the projector augmented-wave method. Phys. Rev. B 59, 1758 (1999).

\section{ACKNOWLEDGEMENTS}

We are grateful to E. A. Kim and S. Y. Park for fruitful discussions. This work was supported by the Institute for Basic Science in Korea (Grant No. IBS-R009-G2). The Advanced Light Source is supported by the Office of Basic Energy Sciences of the U.S. DOE under Contract No. DE-AC02-05CH11231.

\section{AUTHOR CONTRIBUTIONS}

W.K. conceived the work. W.K., Y.K.K., B.K., and C.K. (Chul Kim) performed ARPES measurements with the support from J.D.D., and W.K. and Y.K.K. analyzed the data. W.K., W.J., J.K., and M.K. performed the LEED measurement with support from A.B. Samples were grown and characterized by Y.Y.. C.H.K. led the theoretical study and performed the DFT calculation. All authors discussed the results. W.K. and C.K. (Changyoung Kim) led the project and manuscript preparation with contributions from all authors.

\section{COMPETING INTERESTS}

The authors declare no competing interests.

\section{ADDITIONAL INFORMATION}

Supplementary information is available for this paper at https://doi.org/10.1038/ s41535-020-00306-1.

Correspondence and requests for materials should be addressed to C.K.

Reprints and permission information is available at http://www.nature.com/ reprints

Publisher's note Springer Nature remains neutral with regard to jurisdictional claims in published maps and institutional affiliations.

(i) Open Access This article is licensed under a Creative Commons adaptation, distribution and reproduction in any medium or format, as long as you give appropriate credit to the original author(s) and the source, provide a link to the Creative Commons license, and indicate if changes were made. The images or other third party material in this article are included in the article's Creative Commons license, unless indicated otherwise in a credit line to the material. If material is not included in the article's Creative Commons license and your intended use is not permitted by statutory regulation or exceeds the permitted use, you will need to obtain permission directly from the copyright holder. To view a copy of this license, visit http://creativecommons. org/licenses/by/4.0\%.

(c) The Author(s) 2021 